\title{
Influence of SLES-layered double hydroxides on the mechanical and biodegradation properties of poly (lactic acid) nanocomposites
}

\author{
Siti Hasnawati Jamal ${ }^{1}$ *, Ong Keat Khim ${ }^{1}$, Noor Azilah Mohd Kasim ${ }^{2}$, Mansor Ahmad ${ }^{3}$, Wan Md Zin Wan Yunus ${ }^{2}$ \\ ${ }^{1}$ National Defence University of Malaysia, Department of Chemistry and Biology, Centre for Defence Foundation Studies, Kem Sg. \\ Besi 57000 Kuala Lumpur, Malaysia \\ 2National Defence University of Malaysia, Department of Defence Science, Faculty of Defence Science and Technology, Kem Sg. \\ Besi 57000 Kuala Lumpur, Malaysia \\ ${ }^{3}$ Universiti Putra Malaysia, Department of Chemistry, Faculty of Science, Serdang, 43400 Selangor, Malaysia
}

\section{A R T I C LE IN F O}

Article history:

Received 25 March 2017

Received in revised form

15 August 2017

Accepted 25 September 2017

Keywords:

Poly (lactic acid)

Layered double hydroxides

Nanocomposites

Tensile strength

Biodegradability

\begin{abstract}
A B S T R A C T
Petroleum based polymers have great attention due to its excellent properties. Unfortunately, its uses give detrimental impacts to the health of our environment. Poly (lactic acid), PLA is recognised as one of the potential biodegradable polymers as it exhibits almost similar properties to nonbiodegradable polymers. In this report, nanocomposites composed of PLA and sodium lauryl ether sulphate modified layered double hydroxides, SLESLDH were prepared by solvent casting method to enhance PLA tensile strength. The pristine LDH used was synthesised by a co-precipitation method and then modified by surfactant via an anion exchange process. Xray diffraction result showed LDH interlayer spacing increased from $8.10 \AA$ to $34.23 \AA$ indicated intercalation of the surfactant molecules into LDH was successfully carried out. The result of tensile strength test showed the addition of $1 \mathrm{wt} \%$ of SLES-LDH in the PLA, improved $26 \%$ of its tensile strength compared to that of the pure sample. To study the presence of SLESLDH on the product biodegradability, burial test was carried out. It reveals that PLA nanocomposites degrade faster than the starting polymer and the degradation is SLES-LDH content dependent.
\end{abstract}

(C) 2017 The Authors. Published by IASE. This is an open access article under the CC BY-NC-ND license (http://creativecommons.org/licenses/by-nc-nd/4.0/).

\section{Introduction}

Most commonly used polymers such as polyethylene terephthalate, polypropylene and polystyrene, are petroleum based and nonbiodegradable materials. Therefore, use of these products should be avoided or minimised in order to reduce their impact to environment deterioration. Consequently, biodegradable polymers received a lot of attention and they are good alternatives for the petroleum based materials. Poly (lactic acid), PLA is a thermoplastic polymer which is biodegradable and can be produced by many renewable resources (Anderson et al., 2008) such as corn, potato and sugar beet. Therefore, it is an attractive alternative to conventional petrochemical-based polymers. This polymer degrades naturally and completely into water and carbon dioxides without harmful products (Bordes et al., 2009). In addition, PLA has similar

\footnotetext{
* Corresponding Author.

Email Address: hasnawati@upnm.edu.my (S. H. Jamal) https://doi.org/10.21833/ijaas.2017.012.020

2313-626X/@ 2017 The Authors. Published by IASE.

This is an open access article under the CC BY-NC-ND license

(http://creativecommons.org/licenses/by-nc-nd/4.0/)
}

properties with non-biodegradable polymers. However its high stiffness and brittleness have to be modified to allow it to be used more applications.

One of the approaches to modify the PLA properties to make it more useful industrial applications is to blend it with stearic acid modified LDH (Mahboobeh et al., 2010; Mahboobeh et al., 2012). LDH is anionic clay which consists of positively charged metal hydroxide sheets with exchangeable anions and water molecules. The general composition of LDH can be represented by

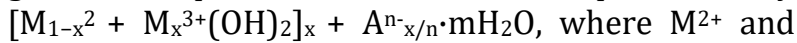
$\mathrm{M}^{3+}$ are divalent and trivalent cations, and $\mathrm{A}^{\mathrm{n}-}$ is an exchangeable anion. This compound has been given much attention recently because it is facile, easy to prepare, as well as environmental friendly (Williams and O'Hare, 2006; Katiyar et al., 2010; Mahboobeh et al., 2012; Livi et al., 2012). Hydrotalcite is naturally occurred LDH (Hochstetter, 1842) but its synthesis was attempted since 1942 (Feitknecht and Gerber, 1942). Modification of LDH by an ion exchange process facilitates intercalation of bulky organic anions into its layers, resulting in enhancement of its hydrophobicity property. It is reported that anionic surfactants with at least one anionic end group and 
long hydrophobic tail are the best compounds to be intercalated resulting an increase of interlayer spacing of LDH which then accommodates intercalation of large species (Maiti et al., 2008; Zhao et al., 2008; Luo et al., 2009). Other approaches include modification by ionic liquids method (Livi et al., 2012) and grafted $\mathrm{TiO}_{2}$ particles (Luo et al., 2009). In this report we describe the results of our attempt to modify LDH by SLES. The product of this modification is then used as an additive for PLA nanocomposites preparation.

\section{Materials and methods}

\subsection{Materials}

Magnesium nitrate hexahydrate and aluminium nitrate nonahydrate (Merck, Germany) were used to synthesis pristine LDH. PLA (Bio-Polymer 3052D) was supplied by NatureWorks LLC, USA. SLES was supplied by Bekee (M) Sdn Bhd, Malaysia.

\subsection{Synthesis of pristine LDH}

Synthesis of LDH was carried out by heating at 70 ${ }^{\circ} \mathrm{C}$, adjusted using $1 \mathrm{M}$ sodium hydroxides solution $500 \mathrm{~mL}$ aqueous solution containing $38.44 \mathrm{~g}$ of magnesium nitrate hexahydrate and $18.76 \mathrm{~g}$ of aluminium nitrate nonahydrate at $100 \mathrm{rpm}$ for 24 hours. The LDH obtained was filtered, washed with deionized water thoroughly and dried at $60{ }^{\circ} \mathrm{C}$ for 24 hours.

\subsection{Modification of LDH by SLES}

SLES-modified LDH was prepared by shaking required amount of LDH in $500 \mathrm{~mL}$ of SLES solution at $75{ }^{\circ} \mathrm{C}$ for 24 hours. The SLES solution was prepared by dissolving the required amount of this compound in $500 \mathrm{~mL}$ water at $50{ }^{\circ} \mathrm{C}$ and $500 \mathrm{rpm}$. The modified LDH was then filtered, washed from several times and dried an oven for 24 hours and 60 ${ }^{\circ} \mathrm{C}$.

\subsection{Preparation of PLA/SLES-LDH Nanocomposites}

PLA and its nanocomposites sheets were prepared using solvent casting method. Ten grams of PLA was first dissolved in $120 \mathrm{~mL}$ chloroform at 40 ${ }^{\circ} \mathrm{C}$. For the composite preparation, a chosen amount of modified LDH was then added into the prepared PLA solution. Then the mixtures were stirred vigorously for 3 hours, sonicated for another 30 minutes, casted in a petri dish and allowed to dry at room temperature until the film was obtained. The amount of the LDH used was 1, 3, 5, 7 and $10 \mathrm{wt} \%$.

\subsection{Characterization}

(a) X-Ray diffraction method: X-ray diffraction patterns of PLA samples were recorded using a
XRD 6000 Diffractometer, Shimadzu. The instrument was operated at $30 \mathrm{kV} / 30 \mathrm{~mA}$ and produced $\mathrm{Cu}-\mathrm{K} \alpha$ radiation $(\lambda=0.15405 \mathrm{~nm})$. All samples were scanned at a rate $0.2^{\circ} 2 \theta / \mathrm{min}$ in a range from 2 to $65^{\circ}$.

(b) Fourier transform infrared spectra: Spectra of samples sheets were recorded using a Perkin Elmer System FTI 1650 Spectrum BX, Spectrometer using an average 10 scan in the range from 400 to $4000 \mathrm{~cm}^{-1}$.

(c) Tensile measurement: Five dumbbell shape samples were prepared using ASTM Standard D638-V. The thickness and width of the gauge section of each specimen were calculated. The test was carried out by Instron Universal Testing Machine to obtain value of tensile strength, tensile modulus and elongation at break.

(d) Biodegradation test: The biodegradation test of the PLA film was carried out by burying the samples in the soil at our campus in the Sungai Besi Camp, Kuala Lumpur Malaysia for six months. Each film sample was cut into $1 \mathrm{~cm} \mathrm{x} 1$ $\mathrm{cm}$ dimension and buried in the soil at a depth of about $8 \mathrm{~cm}$ from the ground surface for a chosen period. It was then washed with distilled water and dried in an oven at $35^{\circ} \mathrm{C}$. The weights before and after the burial of each sample were recorded.

\section{Results and discussion}

\subsection{Characterization of SLES-LDH}

In this work, preparation of LDH was successfully modified by SLES via ion exchange process as shown in Fig. 1. The modification of LDH was conducted by exchanging the original anion, $\mathrm{NO}_{3}^{-}$to long alkyl surfactant anion, $\mathrm{CH}_{3}\left(\mathrm{CH}_{2}\right)_{10} \mathrm{CH}_{2}\left(\mathrm{OCH}_{2} \mathrm{CH}_{2}\right)_{n} \mathrm{OSO}_{3}$ - in order to enlarge the interlayer spacings of $\mathrm{LDH}$, hence enhance its hydrophobicity.

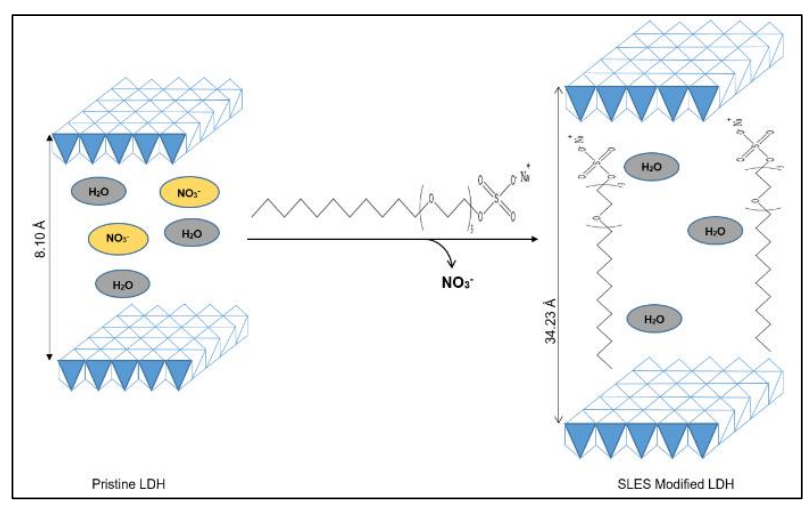

Fig. 1: Schematic representation of ion exchange process

Fig. 2 shows the x-ray diffraction results of pristine LDH and SLES-LDH. Sharp peaks in both pristine $\mathrm{LDH}$ and SLES-LDH pattern indicate that they are crystal with well-defined interlayer structure (Jamal et al., 2015). The peak of SLES-LDH was at $2 \theta$ of $2.58^{\circ}$ which smaller than that of the pristine LDH shows the intercalation of surfactant anions into LDH interlayers was successfully carried 
out; therefore its interlayer distance is bigger than that of the original material. The interlayer spacings can be calculated using Bragg's equation where $\mathrm{n} \lambda=$ $2 d \sin \theta$ where $\mathrm{n}$ is $1, \lambda=$ wavelength of $\mathrm{Cu}-\mathrm{K}_{\alpha}$ radiation, $\mathrm{d}=$ interlayer spacing of pristine $\mathrm{LDH}$ and SLES-LDH and $\theta=$ diffraction angle. The calculation shows the interlayer spacings for the pristine $\mathrm{LDH}$ is 8.10 ̊ while for the SLES-LDH is $34.23 \AA$. Increase of the interlayer spacing due to intercalation of a long hydrophobic anionic surfactant chain has been reported earlier (Maiti et al., 2008).

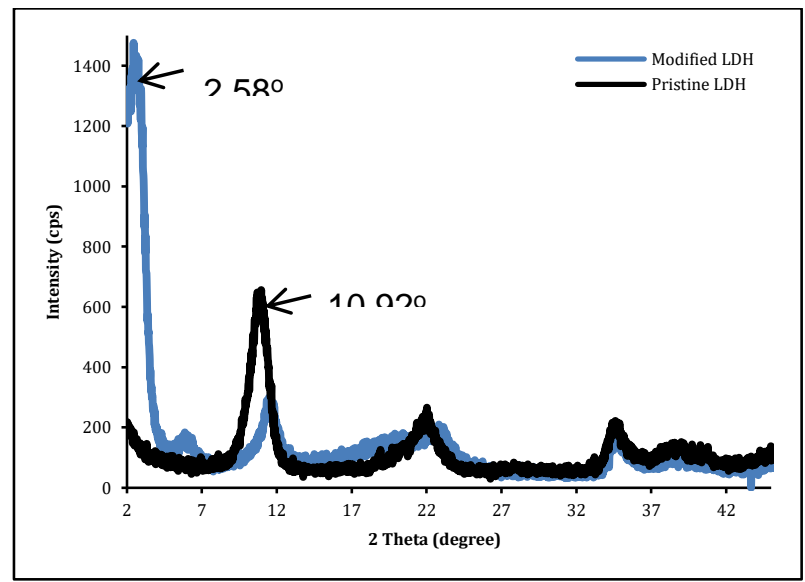

Fig. 2: XRD patterns of pristine and modified LDH

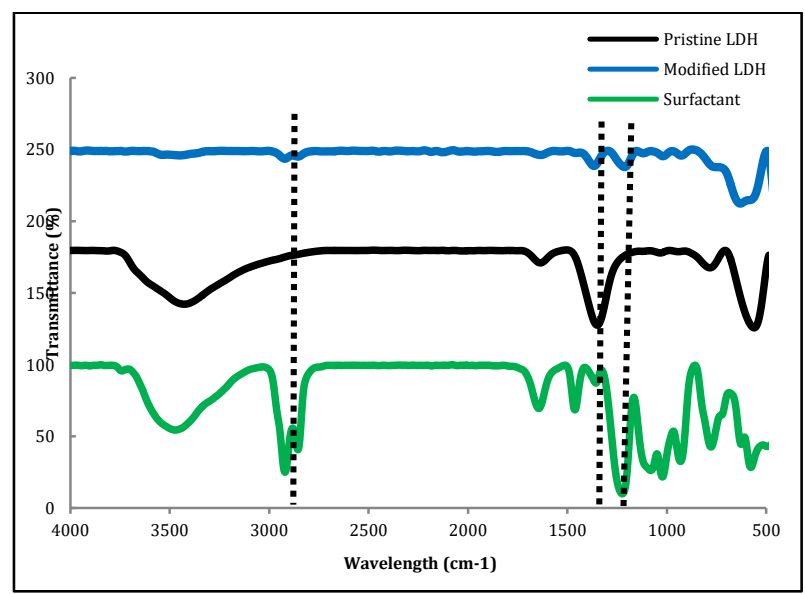

Fig. 3: FTIR spectra of pristine and modified LDH and SLES

Fig. 3 shows FTIR spectra of pristine LDH, SLES and SLES-LDH. Graph indicates pristine LDH does not have three peaks that obviously appeared in SLES-LDH curve.

For pristine $\mathrm{LDH}$, the broad peak at $3439 \mathrm{~cm}^{-1}$ in the pristine LDH spectrum is due to the presence of $\mathrm{O}-\mathrm{H}$ stretch from the interlayer of $\mathrm{LDH}$ and water molecules. Also the sharp peak at $1357 \mathrm{~cm}^{-1}$ shows the existence of nitrate group in the pristine LDH (Wang et al., 2005; Jamal et al., 2015).

For SLES-LDH spectrum, in addition to the presence of LDH major absorption peaks as mentioned above, it also has bands attributed to SLES anion at peak of $2925 \mathrm{~cm}^{-1}$ and $2950 \mathrm{~cm}^{-1}$ which are $\mathrm{C}-\mathrm{H},-\mathrm{CH}_{2}$ and $-\mathrm{CH}_{3}$ absorption bands. Furthermore, $\mathrm{SO}_{3}^{-}$group also appears at $1207 \mathrm{~cm}^{-1}$ in the spectrum designates the existence of SLES anion (Jamal et al., 2015).

\subsection{Characterization of PLA/SLES-LDH nanocomposites}

Tensile strength and tensile modulus and elongation at break of neat PLA and its nancomposites are displayed graphically in Fig. 4 and 5 respectively. Addition of 1 wt $\%$ SLES-LDH in the PLA improves the tensile strength of PLA/SLESLDH nanocomposites by about $26 \%$ which is from 19.0 $\mathrm{MPa}$ to $23.0 \mathrm{MPa}$, and attained the highest strength with $1 \mathrm{wt} \%$ SLES-LDH for PLA (23 MPa). It is reported that dispersion of SLES-LDH in PLA resulted in strong interfacial interaction between polymer matrix and layered silicates, which produces higher mechanical strength when compared to conventional microcomposites (Ren, 2010). However, further increase amount of SLESLDH slightly does not affect the tensile strength which can be considered as no improvement in tensile strength. The addition of SLES-LDH also shows the increment of tensile modulus of PLA nanocomposites compared to neat PLA.

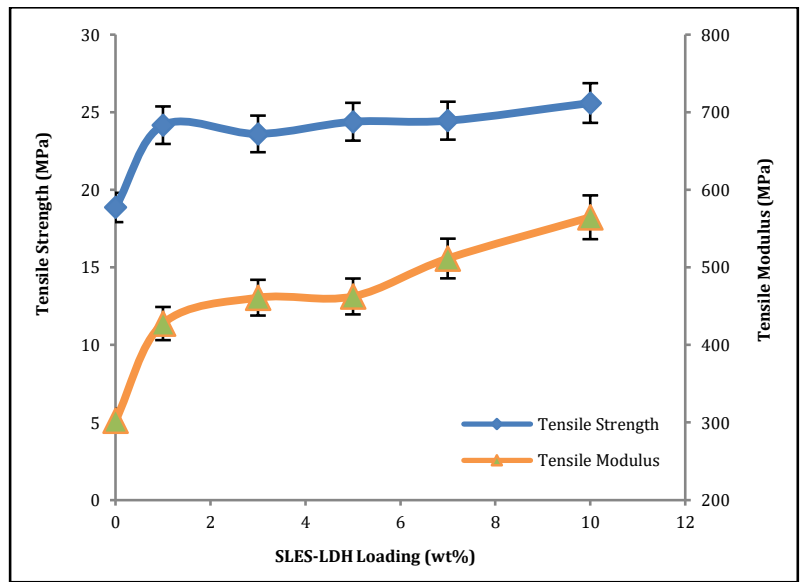

Fig. 4: Effect on SLES-LDH content on tensile strength and tensile modulus of PLA/SLES-LDH nanocomposite

For Fig. 5, the elongation at break of PLA decreases with the addition of SLES-LDH. The presence of SLES-LDH reduced the mobility of PLA chains, thereby reducing the flexibility of PLA. Consequently, higher SLES-LDH loading produced high tensile strength with low elongation.

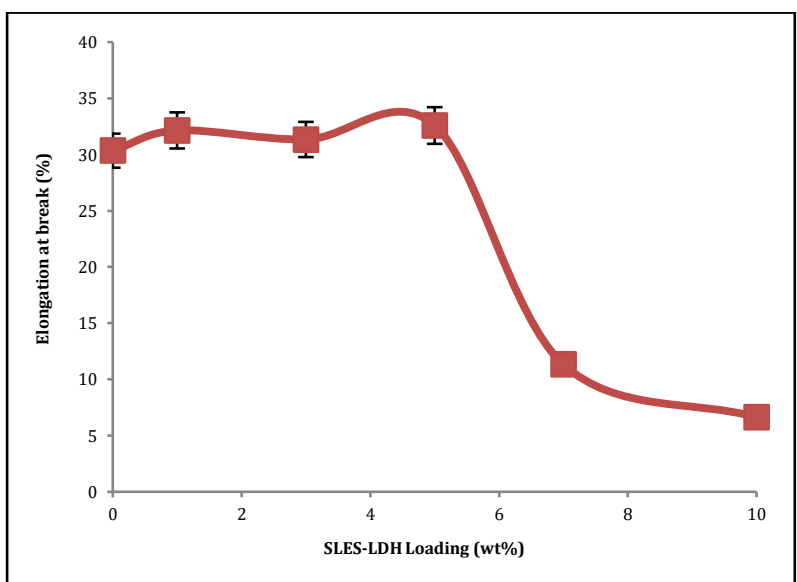

Fig. 5: Effect of SLES-LDH content on elongation at break of PLA/SLES-LDH nanocomposites 
SEM was used to observe surface morphology of the fractured tensile strength samples. Fig. $6 \mathrm{a}$ and $6 \mathrm{~b}$ displays the comparison of surface micrograph of neat and PLA/1wt\% SLES-LDH nanocomposites.

Fig. 6a shows the smooth and brittle surface of neat PLA polymer. In contrast, rough and stretch surface can be seen in PLA nanocomposite fractured surface (Fig. 6b) indicated that strong characteristic in PLA when added SLES-LDH. The addition of SLESLDH improves the interaction between additive and polymer, showed that the nanocomposites are compatible with the SLES-LDH, and resulted in the improvement of tensile strength.

Fig. 7 shows the weight loss percentages of the pure PLA and PLA nanocomposites buried in the soil for up to six months. It displays that degradation rate the composites is higher than that of the pure PLA. It also indicates the weight loss of PLA nanocomposite increases as the weight percentage of SLES-LDH in the composites is enhanced. Similar observations was reported by Mahboobeh et al. (2012) when they studied the degradation of PLA/stearate modified LDH composites and Ray and Okamoto (2003) when the worked with the PLA/modified MMT degradation. These results also reveal that the pure PLA degrades slowly; only about $1 \%$ of weight loss is recorded after 6 months it is buried in the soil. In contrast, the PLA containing 10wt\% SLES-LDH degrades faster whereby $17 \%$ of the weight loss is recorded the same period of the exposure. It is reported that PLA degradation is a two-step reaction. Initially, high molecular weight of PLA is hydrolysed to produce lower molecular weight compounds. This step is temperature and humidity dependent. Then, the degradation process is continued with the presence of microorganism in the environment to convert them into carbon dioxides, water and humus (Tsuji and Ikada, 2000; Gruber and O’Brien, 2002).

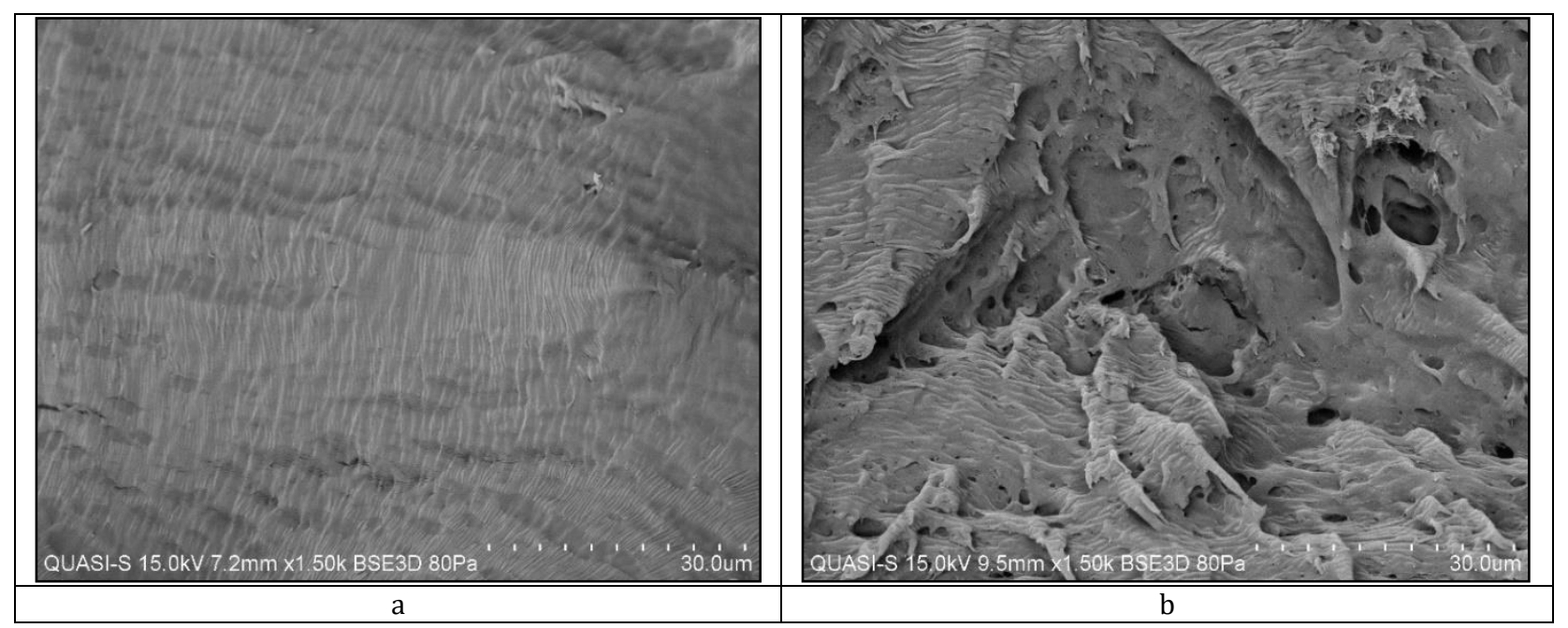

a

Fig. 6: (a) Neat PLA; (b) PLA/1 wt\% SLES-LDH nanocomposite

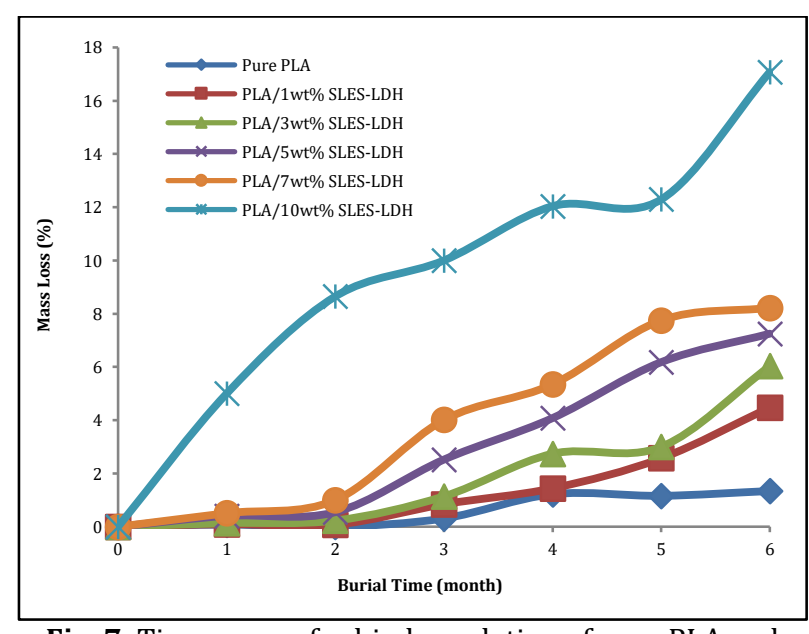

Fig. 7: Time course for biodegradation of pure PLA and PLA nanocomposites with various weight ratios of LDH

\section{Conclusion}

\section{PLA/SLES-LDH} nanocomposites were synthesized successfully via solvent casting method. The used modification of LDH by SLES was prepared by ion exchange method. With the addition of $1 \mathrm{wt} \%$
SLES-LDH in PLA, the enhancement of tensile strength was obtained around $26 \%$ but poor result on its flexibility. For biodegradability study, fast degradation can be obtained in PLA nanocomposites compared to its pure with $17 \%$ mass loss after six months buried in soil. However, the degradation rate depends on many factors such as moisture and temperature.

\section{Acknowledgement}

The work was financially supported by Research Acculturation Grant Scheme (RAGS) (Grant No. RAGS/2012/UPNM/SG01/1) and Fundamental Research Grant Scheme (FRGS) (Grant No. FRGS/1/2013/ST05/UPNM/02/1).

\section{References}

Anderson KS, Schreck KM, and Hillmyer MA (2008). Toughening polylactide. Polymer Reviews, 48(1): 85-108.

Bordes P, Pollet E, and Averous L (2009). Nanobiocomposites: biodegradable polyester/nanoclay system. Progress in Polymer Science, 34(2): 125-155. 
Feitknecht W and Gerber M (1942). Double hydroxides and basic double salts. II Mixed precipitates from calcium-aluminium salts solutions. III Magnesium-aluminium double hydroxides. Helvetica Chimica Acta, 25: 106-131.

Gruber P and O’Brien M (2002). Polylactides “NatureWorksTM PLA. In: Doi Y and Steinbuchel A (Eds.), Bioplymers polyesters III applications and commercial products: 235-249. WileyVCH, Weinheim, Germany.

Hochstetter C (1842). Untersuchung über die Zusammensetzung einiger Mineralien. Advanced Synthesis and Catalysis, 27(1): 375-378.

Jamal SH, Yunus WMZW, Azilah N, Kasimb M, Khima OK, and Ahmadc M, (2015). Preparation and characterization of sodium lauryl ether sulphate modified layered double hydroxides. Jurnal Teknologi (Science and Engineering), 76(3): 109-113.

Katiyar V, Gerds N, Koch CB, Risbo J, Hansen HCB, and Plackett D (2010). Poly L-lactide-layered double hydroxide nanocomposites via in situ polymerization of L-lactide. Polymer Degradation and Stability, 95(12): 2563-2573.

Livi S, Bugatti V, Estevez L, Duchat-Rumeau J, and Giannelis EP (2012). Synthesis and physical properties of new layered double hydroxides based on ionic liquids: Application to a polylactide matrix. Journal of Colloid and Interface Science, 388(1): 123-129.

Luo YB, Wang XL, Xu DY, and Wang YZ (2009). Preparation and characterization of poly (lactic acid)-grafted $\mathrm{TiO}_{2}$ nanoparticles with improved dispersions. Applied Surface Science, 255(15): 6795-6801.

Mahboobeh E, Wan Yunus WMZ, Hussein Z, Ahmad M, and Ibrahim NA (2010). Flexibility improvement of poly (Lactic
Acid) by stearate modified layered double hydroxide. Journal of Applied Polymer Science, 118(2): 1077-1083.

Mahboobeh E, Wan Yunus WMZ, Ibrahim NA, and Shameli K (2012). Degradability enhancement of poly (Lactic Acid) by stearate- $\mathrm{Zn}_{3} \mathrm{Al} \mathrm{LDH}$ nanolayers. International Journal of Molecular Sciences, 13(7): 7938-7951.

Maiti M, Bhattacharya M, Bhowmick AK, (2008). Elastomer nanocomposites. Rubber Chemistry and Technology, 81(3): 384-469.

Ray SS and Okamoto M (2003). Polymer/layered silicates nanocomposites: A review from preparation to processing. Progress in Polymer Science, 28(11): 1539-1641.

Ren J (2010). Biodegradable poly (Lactic Acid): synthesis modification, processing and applications. Tsinghua University Press, Beijing, China.

Tsuji H and Ikada Y (2000). Biodegradable polyesters for medical and ecological applications. Macromolecular Rapid Communications, 21(3): 117-132.

Wang B, Siahaan TJ, and Soltero RA (2005). Drug delivery: Principles and applications. John Wiley and Sons, Hoboken, USA.

Williams GR and O'Hare D (2006). Towards understanding, control and application of layered double hydroxide chemistry. Journal of Materials Chemistry, 16(30): 3065-3074.

Wypch G (2004). Handbook of plasticizers. ChemTech Publishing, Toronto, Canada.

Zhao N, Shi S, Lu G, and Wei M (2008). Polylactide (PLA)/layered double hydroxides composite fibers by electrospinning method. Journal of Physics and Chemistry of Solids, 69(5): 1564-1568. 